\title{
Germanica
}

\section{„Happy New Ears“: Lyrik im Zeichen des „sonic turn“}

Izabela Rakar : "Happy New Ears " : poésie sous le signe du sonic turn

Izabela Rakar: „Happy New Ears": Poetry and the „Sonic Turn"

\section{Izabela Rakar}

\section{(2) OpenEdition}

\section{Journals}

Édition électronique

URL : http://journals.openedition.org/germanica/6868

DOI : 10.4000/germanica.6868

ISSN : 2107-0784

\section{Éditeur}

Université de Lille

\section{Édition imprimée}

Date de publication : 26 juin 2019

Pagination : 117-130

ISBN : 978-2-913857-42-1

ISSN : 0984-2632

\section{Référence électronique}

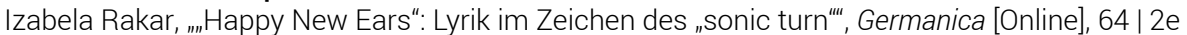
trimestre 2019, Online erschienen am: 01 Januar 2021, abgerufen am 26 Februar 2021. URL: http:// journals.openedition.org/germanica/6868; DOI: https://doi.org/10.4000/germanica.6868 
Tendances 



\title{
„Happy New Ears“: Lyrik im Zeichen des ,sonic turn“"
}

\author{
Izabela Rakar \\ University of Oxford
}

Der „sonic turn“, der im Zug der Sound-Studies-Forschung und künstlerischer Arbeiten aus dem Bereich der Sound Art aufkam ${ }^{1}$, lässt sich in seinen Anfängen auch in der Lyrik-Forschung des 21. Jahrhunderts erkennen. In der US-amerikanischen Forschung erschien 2009 die Aufsatzsammlung The Sound of Poetry/The Poetry of Sound, herausgegeben von Marjorie Perloff und Craig Dworkin, die die enge Verbindung zwischen Lyrik und Klanglichkeit aus unterschiedlichen Perspektiven untersuchte. Mehrere internationale Tagungen widmeten sich in den letzten Jahren den Bedeutungen von Klang2 ${ }^{2}$ In der deutschen Lyrik-Forschung ist Klanglichkeit vor allem in Studien zum Genre der Lautpoesie analysiert und dokumentiert ${ }^{3}$, die akus-

1. - Jim Drobnick, „Listening Awry“, in: Aural Cultures: Sound Art and Society, Banff, Yyz Books, 2004, S. 10.

2. - Zuletzt zum Beispiel die im Oktober 2017 gehaltene interdisziplinäre Konferenz „The Sense of Sound (Le sens du son)“, die Forscher der Französischen Literatur, Musik und Geschichte sowie Klangkünstler zusammenbrachte. URL: https:// as.nyu.edu/content/nyu-as/as/research-centers/maisonfrancaise/events/2017/the-senseof-sound--le-sens-du-son-.html (Zugriff am 27.12.2017).

3. - Siehe Michel Lentz, Lautpoesie/-musik nach 1945: eine kritisch-dokumentarische Bestandsaufnahme, Wien, edition selene, 2000; Christian Scholz, Untersuchungen zur Geschichte und Typologie der Lautpoesie, Obermichelbach, Scholz Verlag, 1989. 
tische Dimension von anderen Formen der Lyrik wird ansatzweise erforscht ${ }^{4}$.

Die deutschsprachige Lyrik der letzten zwei Jahrzehnte kennzeichnet ein Interesse für Klanglichkeit, das mit der Wiederentdeckung der Oralität durch die Lyrik der 1980er und 1990er Jahre sowie der dritten Welle der Lautpoesie, die nach Steve McCaffery in den 1950er Jahren einsetz $^{5}$, nur bedingt zu tun hat. Eine Vielzahl verschiedener Ansätze, Herangehensweisen und Anknüpfungspunkte kennzeichnet diese ,klangorientierten' oder ,klangfokussierten' Arbeiten, die sich nicht immer in das Genre der Lautpoesie einordnen lassen. Dieser Aufsatz untersucht Arbeiten dreier Lyriker, die in diesem Kontext drei unterschiedliche Arbeitsweisen und Positionen, und damit drei zeitgenössische Möglichkeiten der Arbeit mit Klanglichkeit aufweisen. Die Texte der in Berlin lebenden schwedisch-finnischen Lyrikerin und Künstlerin Cia Rinne $(* 1973)$ stellen eine klangorientierte Form der multilingualen Poetik vor, getrieben von klanglichen Assoziationen zwischen verschiedenen europäischen Sprachen. Die lautpoetischen Arbeiten von Valeri Scherstjanoi $(* 1950)$ beziehen sich am stärksten auf das Genre der Lautpoesie, das weiterhin als wichtige Quelle der Beschäftigungen mit Klanglichkeit zu sehen ist. Die multimedialen Arbeiten des digitalen Lyrikers und Programmierers Jörg Piringer (*1974) zeigen das Potential der digitalen Technologie bei der Arbeit mit Klanglichkeit und Stimme. Klanglichkeit ist in allen diesen Fällen kein autonomes Element, sondern wird eng mit textuellen, visuellen und technischen Aspekten verbunden, die im Folgenden analysiert werden sollen.

\section{Cia Rinne: die Klanglichkeit der Sprachen}

Cia Rinnes Interesse für die Klanglichkeit begann als Weiterentwicklung einer visuell-orientierten Poetik: als sie die Verleger ihres ersten Bandes zaroum (2001) baten, aus dem Band vorzulesen, entdeckte sie, dass die Texte voller grafischer Elemente wie z.B. Kästchen, Zeichnungen und Auswahlfragen wider Erwarten auch in der Form der Lesung gut funktionierten ${ }^{6}$. Rinnes zweiter Band

4. - Siehe Britta Herrmann, Dichtung für die Ohren: Literatur als tonale Kunst in der Moderne, Berlin, Vorwerk 8, 2015; Anna Bers, Peer Trilcke, Phänomene des Performativen in der Lyrik. Systematische Entwürfe und historische Fallbeispiele, Göttingen, Wallstein, 2017; Claudia Benthien, Performing Poetry. Mediale Übersetzungen und situationale Rahmungen zeitgenössischer Lyrik (Forschungsprojekt: 2015-17). Meine Forschung zum Aspekt der Klanglichkeit in der zeitgenössischen Lyrik wurde durch die Alexander von Humboldt-Stiftung ermöglicht, bei der ich mich hiermit sehr bedanke.

5. - Steve McCaffery, ,Sound Poetry - A Survey“. http://www.ubu.com/papers/ mccaffery.html (Zugriff am 29.11.2018).

6. - Maintenant \#52: Cia Rinne. Ein Interview mit Cia Rinne von SJ Fowler. http://www.3ammagazine.com/3am/maintenant-52-cia-rinne/ (Zugriff am 2.1.2018). 
notes for soloists (2009) ist in hohem Maße von dieser Entdeckung angeregt und orientiert sich viel stärker an der Klanglichkeit der Texte - eine Entwicklung, die also zum Teil als Folge der Anforderungen von Veranstaltern und des Lesemarketings zu sehen ist, zum Teil aber auch aus der Poetik selbst hervorgeht. Obwohl notes for soloists in serieller Folge sehr unterschiedliche Texte versammelt, entfaltet sich das poetische Potential dieser Texte oft von der „Evokalisierung“ her, die laut Garrett Stewart nicht nur das Hervorrufen der Mündlichkeit bezeichnet, sondern auch „Auralität“, d.h. die vom Text hervorgerufene Klanglichkeit ${ }^{7}$. Rinnes Wortfolgen sind in gewisser Hinsicht bereits das Produkt der destabilisierenden Bewegung zwischen dem graphischen Text und der auralen Evokalisierung:

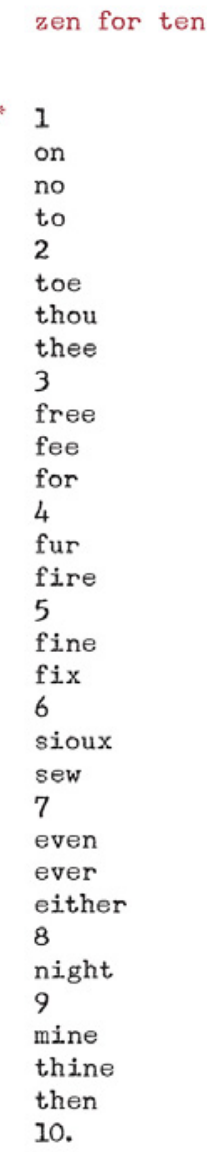

7. - Garrett Stewart, Reading Voices: Literature and the Phonotext, Berkley, University of California Press, 1990. 
„zen for ten" 8 kennzeichnet die akustische Herangehensweise an das Aufzählungssystem und seine Zeichenfolge, die mit vom Klang her möglichst nahen Wörtern (beinah Homophonemen) kombiniert wird. Was dieses serielle Schreibverfahren mit der Zen-Philosophie gemeinsam hat, ist u.a. der Verzicht auf die Subjektivität des Autors, die hinter die Logik der Aufzählung und der Klangassoziationen zurücktritt - zurecht wurde Rinnes Band auch als Beispiel der konzeptuellen Lyrik rezipiert und anthologisiert ${ }^{9}$.

Über die Ähnlichkeiten in der Klanglichkeit wechseln die Texte nicht nur zwischen dem numerischen und dem alphabetischen System, sondern auch zwischen verschiedenen Sprachen, vor allem zwischen dem Englischen, Deutschen, Französischen und Italienischen ${ }^{10}$. Die Bedeutung der Wörter ändert sich jedoch nicht grundlegend: semantische Verschiebungen sind möglich vor allem wegen der klanglichen Nähe zwischen den europäischen Sprachen der gleichen Sprachfamilie oder aufgrund des Kontaktes zwischen den Sprachen. Im folgenden Text ermöglicht die klangliche Nähe eine Reihe von graduellen Verschiebungen: vom französischen „l'or“ (Gold), zum italienischen „loro“ (sie), zur französischen Variante (,leur"), zu „l'heure“ (Zeit) bis hin zum „l'horreur“ (Horror):

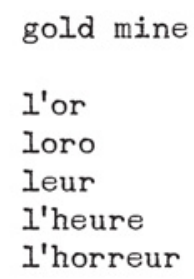

Die einzelnen Texte basieren auf dem Prinzip der Wiederholung und der Variation - auf der phonemischen, morphemischen, aber auch syntaktischen Ebene - indem sie die gleichen Sätze bzw. Satzteile wiederholen und variieren. In den Ähnlichkeiten in der Verfahrensweise sowie dem erzielten Effekt lassen sich einige Parallelen zur Neuen Musik sehen, vor allem zur Minimal

8. - Cia Rinne, notes for soloists, OEI Editör, Stockholm, 2009 (sowie: Le clou dans le fer, Reims 2011; kookbooks, Berlin 2017; Gyldendal, Kopenhagen 2018). Die beiden folgenden Texte ebd.

9. - Marjorie Perloff, „Towards a conceptual lyric: From Content to Context“, Jacket 2 (28. Juli). http://jacket2.org/article/towards-conceptual-lyric (Zugriff am 14.05.2019), sowie I'll Drown My Book: Conceptual Writing by Women, hrsg. von Caroline Bergvall et al., Los Angeles, Les Figues Press, 2012.

10. - Für den Aspekt der Mehrsprachigkeit siehe Markus Huss, Julia Tidigs, „The Reader as Multilingual Soloist: Linguistic and Medial Transgressions in the Poetry of Cia Rinne", in: Borders under Negotiation: VAKKI Symposium XXXV. Vaasa 12.-13.2.2015, hrsg. von D. Rellstab, N. Siponkoski, Vaasa, VAKKI Publications, 2015, S. 16-24. 
Music, die Rinne zu einem ihrer Inspirationsbereiche zählt ${ }^{11}$. Die Wiederholung der sich langsam ändernden musikalischen Einheiten ähnelt der Wiederholung von linguistischen Einheiten, die allmählich Bedeutungsverschiebungen erzeugt:

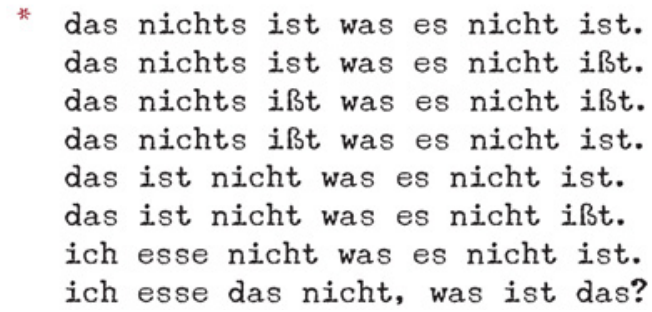

Über das Spiel mit den Homonymen ist/isst wird aus einem philosophischen Satz (,das nichts ist was es nicht ist“) eine alltägliche Aussage über das Essen. Texte, die stark mit Wiederholung arbeiten, gründen auf einem sehr konsistenten Rhythmus, der den Rahmen für die graduellen Bedeutungsverschiebungen formt. Ähnlich wie in der Minimal Music rückt die meditative Art des Wiederholungsprinzips den Fokus auf die Materialität des Werkes, seine kleinsten Bestandteile und subtilere Komplexitäten.

Darüber hinaus beschäftigt sich Rinne mit Klanglichkeit nicht nur auf textueller Ebene, sondern auch als akustisch-räumliches Phänomen in Form von Klanginstallationen. Die erste Solo-Ausstellung Sounds for Soloists im Grimmmuseum 2012 zeigte die Klanginstallationen notes for telephone und sans y/eux, die beide auf aufgenommenen Texten von Rinne basierten: notes for telephone verwendete z.B. ein altes Telefon mit Wählscheibe als Vermittlungsmedium der Texte, während sans y/eux als räumliche Installation mit sieben Lautsprechern positioniert im Halbkreis konzipiert war. Parallel zu Rinnes Annäherung an das analoge Medium der Schreibmaschine, das das visuelle Bild der Texte bestimmt, geht hier auch die Beschäftigung mit Klanglichkeit in eine ähnliche Richtung - nämlich der Rematerialisierung als Gegenbewegung zur Digitalisierung von Klang, der immer und überall abrufbar geworden ist. Vor allem im Format der Lesung wird Klanglichkeit zu einem einmaligen und unwiederholbaren Ereignis, aber auch in diesen Installationen geschieht etwas Ähnliches: der Klang wird in einem konkreten, musealen Kontext (der Ausstellung) lokalisiert und an ein Objekt gebunden. Im Gegensatz zu den Kopfhörern oder Lautsprechern ist das Telefon noch stärker ein Medium der

11. - Siehe Cia Rinne für Full Stop Magazine, im Gespräch mit dem Herausgeber Michael Schapira. http://www.full-stop.net/2016/06/28/interviews/michael-schapira/ cia-rinne/ (Zugriff am 1.2.2018). 
Kommunikation, des Dialogs und der Präsenz. Das bereits historisch gewordene Medium des analogen Telefons deutet die Kommunikation zwischen genau zwei Personen an, in diesem Fall der Lyrikerin und der Rezipientin, und eröffnet dadurch die nur in diesem Medium realisierbare individuelle Rezeption von Lyrik als akustisches Ereignis, das im Gegensatz zu einer digitalen Audiodatei nicht immer und überall abrufbar ist. Es geht dabei nicht um eine unkritische Nostalgie für das Analoge, vielmehr ist es eine Poetik, die sich zwischen analogen und digitalen Medien bewegt ${ }^{12}$ und dabei das jeweils idiosynkratische Moment des Mediums festzuhalten versucht. Die Poetik lässt sich in Zusammenhang mit Überlegungen zum Hören an der Schnittstelle zwischen Analogem und Digitalem kontextualisieren, wie zum Beispiel Damon Krukowskis The New Analog: Listening and Reconnecting in a Digital World (2017).

\section{Valeri Scherstjanoi: das unendliche Spektrum der physisch produzierten Laute}

Während Rinnes Einflüsse vor allem aus der Fluxus-Kunst und Musik kommen ${ }^{13}$, bezieht sich Valeri Scherstjanois Arbeit im Vergleich $\mathrm{zu}$ anderen klangorientierten Lyrikern am stärksten auf das Genre der Lautpoesie. Die Aufsatzsammlung Mein Futurismus fokussiert verschiedene russische und deutsche Autoren, die für Scherstjanois poetische Praxis bedeutend sind: Wladimir Majakowski, Daniil Charms, Kurt Schwitters, Wassily Kandinsky, Filippo Tommaso Marinetti, Welimir Chlebnikow, Krutschonych und Carlfriedrich Claus - Lyriker, die mit dem russischen Futurismus, deutschem Dadaismus und der Wiederentdeckung der Lautpoesie in den 1950er und 1960er Jahren zu verbinden wären, dem Genre der „sinnfreie[n] Dichtung aus Geräuschen, Sprachlauten und Lautfolgen"14. Als Vertreter der Lautpoesie und Nachfolger des Futurismus nimmt Scherstjanoi im deutschsprachigen Kontext, wo es seit der Wiederentdeckung des Genres in den 1950er und 1960er Jahren durch Lyriker wie Ernst Jandl, Gerhard Rühm, Oskar Pastior und Franz Mon keine direkte Anknüpfung gab, eine besondere Position ein. Die Poetik setzt sich mit der Frage auseinander, wie ein Genre, das „von der Zeit abgestempelt“ zu sein scheint ${ }^{15}$, fortgesetzt werden könnte. Scherstjanoi will sagen, dass die Lautpoesie für den Menschen und seinen Artikulationsapparat

12. - Es gibt von Rinne ebenso das auf Youtube abrufbare Klangstück sounds for soloists (2012).

13. - Cia Rinne für Full Stop Magazine, a.a.O.

14. - Valeri Scherstjanoi, Mein Futurismus, Berlin, Matthes \& Seitz, 2011, S. 143.

15. — Ebd., S. 151. 
etwas Primäres ist, eine Art Quelle, die jederzeit durch ein artikulierendes, auf die Umwelt reagierendes Subjekt belebt werden kann. Zugleich aber wird klar, dass diese Poetik sehr stark aus den Quellen der Lautpoesie schöpft und sich auch dadurch, im Akt der forschenden, recherchierenden Auseinandersetzung fortsetzt.

Vom Nachtalpenweg (2009), entstanden in Zusammenarbeit mit Mara Genschel, widmet sich den Glossolalien als vergessenen Quellen der Lautpoesie. Als Buch und CD steht Vom Nachtalpenweg an der Schnittstelle zwischen Schrift und mündlicher, akustischer Interpretation eines ursprünglich mündlichen Phänomens. Glossolalie bezeichnet den Akt des flüssigen Sprechens, das Aspekte wie Tonfall, Rhythmus, Intonation und Pausen beinhaltet, um die Rede in kleinere Einheiten zu teilen. Jede Einheit besteht aus Silben, die aus Konsonanten und Vokalen einer dem Sprecher bekannten Sprache geformt werden, doch als bloße „Fassade der Sprache“ bleibt das Sprechen grundsätzlich unverständlich ${ }^{16}$. Laut de Certeau ist Glossolalie für mündliche Kommunikation das, was die Utopie für den sozialen Raum ist: in Form des linguistischen Simulakrums umschließt sie alles, was Sprache nicht ist und wird dennoch von einer sprechenden Stimme hervorgebracht ${ }^{17}$. Das Interesse an Glossolalien als Formen mündlicher Utopie ist mit den Avantgarden des 20. Jahrhunderts und der Geschichte der Lautpoesie eng verbunden: Der radikale Bruch mit der konventionellen Sprache ging Hand in Hand mit der Suche nach einer arationalen Sprache und Rekonzeptualisierung von Lyrik als ritueller Praxis, vor allem durch Welimir Chlebnikow und Hugo Ball, die sich beide - worauf Scherstjanoi hinweist - von Glossolalien inspirieren ließen. Vom Nachtalpenweg ist in erster Linie ein historisierendes Projekt, das den Zusammenhang zwischen den historischen Avantgarden (Futurismus, Dada) und der Rezeption von Glossolalien (vor allem aus dem 19. Jahrhundert) herstellt, um sich dann der akustischen Interpretation und Produktion von Texten, die ,im glossolalischen Sinne“ interpretiert werden können, zu widmen.

\section{Friss fifk}

Vom Nachalpenweg in den Schwarzelfenweg über den Gnomenplatz und zurück

Fkfu

Fkfu ufif kufk

n.........fefe

16. - Siehe William J. Samarin, Tongues of Men and Angels: The Religious Language of Pentecostalism, New York, Macmillan, 1972.

17. - Michel de Certeau, „Vocal Utopias: Glossolalias“, Representations, 56/1996, S. 29-47, hier S. 31. 


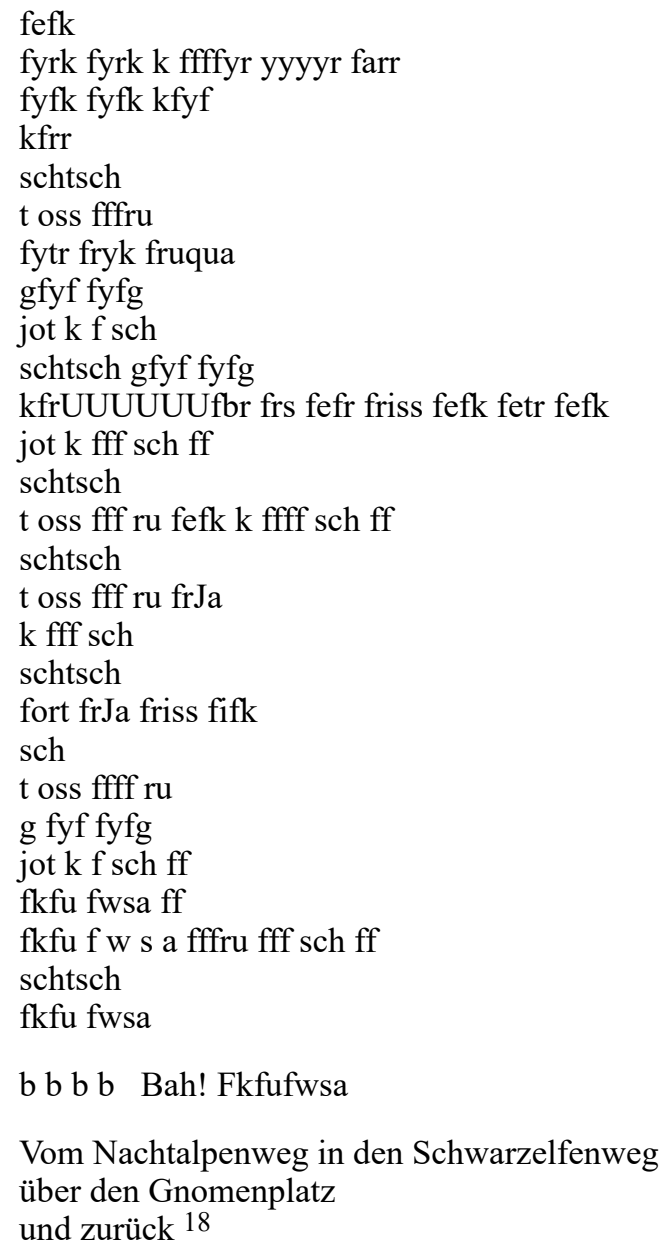

„Friss fifk“ ist eigentlich kein glossolalisches Gedicht: zum einen wegen des verbal-semantischem Anfangs und Endes, zum anderen wegen der hohen Konsonantendichte, die den Eindruck einer silbenbasierten Sprache verhindern. Dennoch finden sich auch in diesem Text typische Elemente von glossolalischen Texten und der Lautpoesie allgemein: an mehreren Stellen findet man tatsächliche deutsche Worte oder verfremdete Fragmente davon, z.B. „fort" (Zeile 25), „friss“ (Zeile 17), „farr" (Zeile 8) - vielleicht als Fragment des Wortes „Fahrrad“-sowie ,jot“ (Zeile 15) und „Ja“ (Zeile 22). Auch der wortbasierten, Glossolalien ähnlichen Lautpoesie gelingt der Bruch mit der

18. - Valeri Scherstjanoi, Mara Genschel, Vom Nachtalpenweg, Berlin, HybridenVerlag (unpaginiert). 
Sprache nie vollkommen, sie inkorporiert immer wieder semantische Reste bekannter Sprachen. Die verstreuten verbal-semantischen Wortfragmente suggerieren vor allem Bewegung. Anders als Rinnes Texte, die von der Klanglichkeit der einzelnen Wörter ausgehen, arbeitet aber „Friss fifk“ mit Klanglichkeit auch auf der Mikroebene der einzelnen Buchstaben und Aneinanderreihungen von Buchstaben bzw. phonetischen Einheiten. Wegen der vielen Konsonanten erinnert der Text stärker an die Lautpoesie von Kurt Schwitters und Raoul Hausmann als an die glossolalischen Lautgedichte Hugo Balls.

Allein durch das Lesen erschließt sich die Bedeutung dieser phonetischen Einheiten nicht, das Gedicht ist, so wie die meisten Texte von Scherstjanoi, ein gutes Beispiel dessen, was Kostelanetz „text-sound text" nennt, ein Text, der akustisch realisiert und gehört werden muss im Gegensatz zu Texten, die visuelle Rezeption voraussetzen sowie akustischen Ereignissen, denen kein Text vorausgeht ${ }^{19}$. Erst durch das Hören des Textes, vorgetragen vom Autor selbst, erschließt sich die Bedeutung des mittleren Teils von „Friss fifk“: Es spricht auf nichtverbaler Ebene von der Fahrradfahrt „Vom Nachtalpenweg in den Schwarzelfenweg / über den Gnomenplatz / und zurück“", indem er unterschiedliche Geräusche fokussiert: zum einen die Geräusche des fahrenden Subjekts, die unterschiedlichen Atemgeräusche - das Pfeifen, Keuchen, Luftschnappen und Entspannen (,fkfu ufif“, „fff“, ,t oss“, ,,jot k f“, ,UUUUUU“), sowie die Fahrgeräusche des Fahrrads (,fyrk“, „fytr fryk“, „sch“, „schtsch“). Der häufigste Laut dieses Gedichts ist der stimmlose Frikativ [f], bei dessen Artikulation eine Engstelle gebildet wird, durch die die ausströmende Luft gezwängt wird. Durch die Kombination der gezwängten f-Laute einerseits und der Sibilante (,sch“) sowie der U-Vokale („UUUUUU“) andererseits entsteht eine lautliche Dynamik, die die Prozesse der Anund Entspannung materialisiert. Den Anfang und das Ende umrahmt eine Wegbeschreibung, die auf konkrete Straßen- und Ortsnamen in Berlin-Weißensee und damit die Fahrradfahrt referiert: die Namen rufen den Bereich des Übernatürlichen hervor, der eng mit dem Phänomen der Glossolalien verbunden ist, aber auch die Alpen, den mitteleuropäischen Raum, der - wie Scherstjanoi hinweist - mit dem Auftauchen von Glossolalien im 19. Jahrhundert verbunden sei ${ }^{20}$.

Scherstjanoi bezieht sich mit seiner Arbeit auf die Lautpoesie des frühen 20. Jahrhunderts, wie die einzelnen Arbeiten und Essays nahelegen, doch genauso ausschlaggebend sind die Entwicklungen der

19. - Richard Kostelanetz, „Text-Sound Art: A Survey“, Performing Arts Journal, 2/1977, S. 61-70, hier S. 2.

20. - V. Scherstjanoi, M. Genschel, Vom Nachtalpenweg, a.a.O. (unpaginiert). 
Lautpoesie in der Nachkriegszeit, die bereits erwähnte dritte Phase der Lautpoesie ab den 1950er Jahren ${ }^{21}$. Das Erforschen des enormen Spektrums von körperproduzierten Lauten auf nichtverbaler Ebene (z.B. Grunzen, Heulen, Schreien), die durch Entwicklungen des Tonbandgeräts und des Tonverstärkers aufzeichenbar geworden sind und so zur Ablösung der Lautpoesie vom Wort führten ${ }^{22}$, bildet den historischen Kontext für den mittleren Teil des Gedichts. Die Fixierung findet hier allerdings durch die Verwendung der alphabetischen Zeichen statt, eine Charakteristik, die sich - ähnlich wie bei Rinne - als Gegentendenz zur Digitalisierung und Technologisierung interpretieren lässt.

\section{Jörg Piringer: mediale Sprachklänge}

Rinnes und Scherstjanois klangfokussierte Arbeiten kennzeichnet eine Tendenz zur analogen oder schriftlichen Fixierung der Klanglichkeit - sei es durch das Medium der Schreibmaschine, das analoge Telefon oder auch Handschrift bzw. Zeichnung 23 - die sich nach 2000 als Reaktion auf die Digitalisierung der Arbeitsbereiche entwickelt. Im Bereich der Musik, des Grafikdesigns und der bildenden Kunst ist anhand ähnlicher Arbeiten die Rede von der , post-digitalen Wende“24, womit eine künstlerische Praxis gemeint ist, die im digitalen Feld auf bestimmte abwesende Momente der Materialität und Präsenz zurückgreift. Mit wenigen Ausnahmen erscheint aber Lyrik (sowie Prosa) weiterhin als eine auf Papier gedruckte Kunstform, für die Prozesse der Digitalisierung weniger entscheidend sind und deren Mainstream auf mediale Veränderungen nicht tiefgreifend reflektiert. Wie allerdings die Geschichte der Lautpoesie zeigt, ist Lyrik als akustisches Ereignis sehr eng mit technologischen Entwicklungen verbunden. Als wichtigste zeitgenössische Beispiele für die Veränderungen der Schreibpraxis durch digitale Medien sind vor allem im nordamerikanischen Kontext die Arbeiten aus dem Umfeld des konzeptuellen Schreibens, der Flarf-Poesie und digitalen Poesie zu nennen. Sie greifen auf heute verfügbare textuelle Materialien im Internet zurück, wobei das Schreiben nicht mehr als Produktion eines noch nicht existierenden Textes begriffen wird, sondern vielmehr als Prozessualisierung, Recycling oder Appropriation von etwas bereits Existierendem. Kenneth Goldsmith eröffnet seine Poetik des ,uncreative writing" mit der folgenden Paraphrase (des Konzeptkünstlers Douglas

21. - Steve McCaffery, „Sound Poetry - A Survey“, a.a.O.

22. - Ebd.

23. - Für die Fixierung der eigenen Lautpoesie entwickelte Scherstjanoi auch ein eigenes Notationssystem, scribetische Zeichen, die aus Elementen des lateinischen und russischen Alphabets sowie erfundenen Zeichen bestehen.

24. - Siehe David Berry, Michael Dieter (Hrsg.), Postdigital Aesthetics: Art, Computation and Design, Basingstoke, Palgrave Macmillan, 2015. 
Huebler): „The world is full of texts, more or less interesting; I do not wish to add any more." 25 Die klangorientierten Arbeiten Jörg Piringers wären mit „,The world is full of sounds, more or less interesting; I do not wish to add any more" einzuführen.

Im 21. Jahrhundert scheint das Spezifische an der akustischen Wahrnehmung das unendliche Spektrum der technisch produzierten Klänge und deren komplette Durchdringung des Alltags zu sein, die $\mathrm{zu}$ einer neuen Auseinandersetzung mit Klanglichkeit anhand der Techniken vor allem aus dem Bereich der elektronischen Musik führt - eine Richtung, die nur sehr langsam und ansatzweise für die Lyrik produktiv rezipiert wird. Die ersten elektroakustischen Herangehensweisen an die Lautpoesie in den 1950er Jahren, insbesondere in Frankreich mit François Dufrêne, Bernard Heidsieck und Henri Chopin, entstehen in zeitlicher und räumlicher Nähe zu Entwicklungen in der elektronischen Musik in den 1940er und 1950er Jahren ${ }^{26}$. Dieses Arbeiten mit dem Tonband, Tonbandgerät, Mikrophon und den Verstärkern stellt im Vergleich zur digitalen Klangbearbeitung einen physischen, taktilen Prozess dar, doch das Experimentieren bleibt arbeitsaufwendig und beschränkt, viele Aspekte wie die Geschwindigkeit und Tonhöhe einer Stimme oder die Isolation von Satzfragmenten können nicht in Echtzeit geändert werden. Durch die technologischen Innovationen entwickelt sich das Genre der elektronischen Musik von einem marginalisierten Medium der künstlerischen Avantgarde zum Mainstream ${ }^{27}$. Nach 2000 kommt es zu einem massiven Anstieg von selbstproduzierter elektronischer Musik, die über das Internet öffentlich zugänglich wird. Einfache Audiosoftwares wie z.B. Audacity, anhand derer man die Tonhöhe oder die Geschwindigkeit der Stimme ändern, verschiedene Effekte hinzufügen und Geräusche sampeln kann, sind heute im Internet jedem zugänglich. Lyriker, die diese Entwicklungen wahrgenommen und mitreflektiert haben, die die Verfahren der elektronischen Musik in ihre Arbeitspraxis inkorporiert haben, gibt es allerdings wenige.

Die lange Orientierung der Lyrik an älteren Musikgenres, die immer die Authentizität des Lyrik-Performers herstellen, kritisiert Christian Bök in „When Cyborgs Versify“, wenn er bemerkt: ,,[..] Jazz ist für mich dennoch zu einem nostalgischen, wenn nicht antiquierten Paradigma

25. - Kenneth Goldsmith, Uncreative Writing. Managing Language in the Digital Age, New York, Columbia University Press, 2011, S. 1.

26. - Siehe Larry Wendt, „Sound Poetry: I. History of Electro-Acoustic Approaches II. Connections to Advanced Electronic Technologies“, Leonardo, 18/1985, S. 11-23, Thomas Holmes, Electronic and Experimental Music, Abingdon-on-Thames, Routledge, 2012.

27. - Siehe T. Holmes, Electronic and Experimental Music, a.a.O. 
geworden. Wir haben die poetische Version dieser älteren Impro Form schon geschaffen, aber wir können uns noch nicht so leicht eine Poetik vorstellen, die mit dem neueren Techno Beat verwandt ist.“28 Dabei benutzt Bök selber die elektronische Musik bloß als Inspiration für eine ausschließlich körperproduzierte Form von Lautpoesie, die mit „Synth Loops" ${ }^{29}$ die mittelalterliche Form des Rondos wiederherstellt. Jörg Piringers akustische Arbeiten sind mit der elektronischen Musik noch näher verwandt. Sie bewegen sich an der Grenze zwischen Lautpoesie, Radiokunst, elektronischer Musik und Performance, in einem transdisziplinären Bereich, der auch für die Lautpoesie der 1950er und 1960er Jahre charakteristisch war. Vokàl beispielsweise ist ein Album, das in das Ambient Genre eingeordnet wird und ausschließlich aus gesampelter gesprochener Sprache besteht, die sich vom Klang her von elektronischer Musik kaum unterscheidet. Auch Arbeiten wie das Stück Radioart wieder. sprechen. lernen (2001) greifen auf die Techniken der elektronischen Musik zurück, um dabei stärker die sprachlichen Strukturen und Gesten in den Vordergrund zu rücken.

Diese Arbeiten stellen die Idee des selbstschaffenden, vom Gefühl her geleiteten Geniekünstlers am stärksten infrage. wieder. sprechen. lernen ${ }^{30}$ besteht ausschließlich aus Samples von österreichischen Radionachrichten: aus Schnipseln von Weltnachrichten, Werbung, Sport, politischen Diskussionen, Klängen und Geräuschen. Stimmen bzw. Geräusche werden isoliert, nach bestimmten Mustern arrangiert und neu geordnet. Es entstehen Blöcke der nacheinander geschnittenen Steuerprozentzahlen, Temperaturangaben, Staatennamen oder auch Schreie, die nicht mehr faktische Informationen vermitteln, sondern Klangeigenschaften bzw. Satzteile auf abstrakterer Ebene kondensieren. Durch die Isolation, Wiederholung und technische Bearbeitung von einzelnen Elementen wird der narrative Zusammenhang der Nachrichten durchbrochen und eine neue Bedeutungsebene aufgemacht. Ebenso wie um ein neues "Sprechen lernen“ geht es auf Rezeptionsebene um ein neues „Hören lernen“, ein deautomatisiertes Hören von Nachrichten voller Verfremdungen und Störungseffekte. Die Verlangsamung der Stimme für Katzenfutterwerbung verstärkt z.B. den

28. - Deutsche Übersetzung durch die Verf. (,[...] jazz for me has nevertheless become a nostalgic, if not antiquated, paradigm. We have already created a poetic version of this older, improv form, but we cannot readily imagine a poetic cognate for a newer, techno beat.“). Christian Bök, „When Cyborgs Versify"e, in: Marjorie Perloff, Craig Dworkin (Hrsg.), The Sound of Poetry/The Poetry of Sound, Chicago, The University of Chicago Press, 2009.

29. - Ebd.

30. - Jörg Piringer, wieder. sprechen. lernen, http://joerg.piringer.net/mp3s/wieder-sprechen-lernen.mp3 (Zugriff am 4.1.2019). 
geschmeidig-schmeichelnden Ton, durch die isolierten Kommentare des Leiters einer politischen Diskussion wiederum wird indirekt die Rücksichtlosigkeit eines der Sprecher deutlich gemacht. Zudem rücken der Rhythmus sowie bestimmte phonetische Charakteristiken von gesprochener Sprache stärker in den Vordergrund. Auf der Ebene der Klänge und Geräusche, aber auch in der Bearbeitung von gesprochener Sprache entsteht eine Art Glitch-Ästhetik ${ }^{31}$, wie sie der Musiker Kim Cascone im Jahr 2000 definiert: als post-digitale Tendenz in der elektronischen Musik, die sich gegen den ,sauberen Klang der digitalen Musikproduktion mit Techniken der Dekonstruktion richtet. Indem es die Sprache in ihre kleinsten Partikel schneidet, bewegt sich auch Piringers Stück zwischen den Polen des „delicate“ und „damaging“"32, zwischen der konstruktiven und destruktiven Seite der digitalen Arbeit mit Klang. Experimente mit dem Medium des Radios sind keinesfalls neu und nicht auf digitale Tonbearbeitung beschränkt: mit dem Radiomaterial experimentierte z.B. in den 1990er Jahren unter dem Künstlernamen People Like Us die britische MultimediaKünstlerin Vicky Bennett, indem sie das Material anhand eines DualTape-Decks edierte ${ }^{33}$. Doch die Möglichkeiten der Klangbearbeitung sind bei einem Kassettenrekorder beschränkt. Der Computer eröffnet nicht nur wesentlich mehrere und schnellere Möglichkeiten der Klangbearbeitung, sondern auch die Möglichkeit der Arbeit mit kleineren Klangsegmenten, die Ebene des Mikroklangs beispielsweise, die erst mittels digitaler Technologien hörbar und bearbeitbar wird ${ }^{34}$.

Die drei Beispiele zeigen unterschiedliche Ebenen der Arbeit mit Klanglichkeit: die verbal-semantische Wortebene, die nichtverbale, submorphemische Ebene der physisch produzierten Laute und die Ebene der digital prozessierten Sprache und Klänge. Diese Arbeiten schöpfen aus der Nähe zur Musik, zur Neuen Musik und zur elektronischen Musik, sowie aus der Geschichte der Lautpoesie. Alle klangorientierten Arbeiten positionieren sich gegenüber der Präsenz der digitalen Technologien mit einer postdigitalen Ästhetik, die entweder auf Formen der Materialität und der Präsenz zurückgreift, oder die mittels digitaler Audio-Tools die akustische Oberfläche dekonstruiert. Die paradoxe Materialität der Klanglichkeit kommt in allen Arbeiten zum Ausdruck: indem Klang an ein Objekt gebunden wird, durch

31. - Siehe Kim Cascone, „The Aesthetics of Failure: 'Post-Digital' Tendencies in Contemporary Computer Music", Contemporary Computer Music Journal, 24/2000, S. $12-18$.

32. - Ebd.

33. - Siehe http://discrepant.bandcamp.com/album/early-radio-works-vol1 (Zugriff am 4.1.2019); Ken Hollings, „Cutting Up the Cut Up“e, BBC Radio 4, 25.06.2015.

34. - Siehe Curtis Roads, Microsound, Cambridge, MIT Press, 2004. 
performative Praxis, die die Physikalität des Sprechens hörbar macht, sowie durch digitale Bearbeitung von Stimmen. Die Tendenz und Herausforderung dieser Arbeiten liegt weiterhin in der Erweiterung der Wahrnehmung von Klanglichkeit und der Sprache - auf eine Art, die auch im 21. Jahrhundert mit John Cages Erweiterung der Wahrnehmung und des Denkens über Musik verwandt bleibt. Sie öffnet die Lyrik für neue Geräusche und Arten der Bearbeitung von Sprache, die unsere akustische Wahrnehmung des Alltäglichen verändern. 